Sandra Leeb

\title{
LONG-COVID - EFFIZIENTE BEHANDLUNGS- STRATEGIEN MIT AKUPUNKTUR UND HOMÖOPATHISCHER UNTERSTÜTZUNG
}

\section{Long-COVID - Efficient Acupuncture Strategies Supported by Homeopathic Treatment}

\section{Zusammenfassung}

Der Begriff Long-COVID bezieht sich auf die Symptomatik, die 4 Wochen nach der Infektion weiterhin besteht. Neben Husten und Atemnot fühlen sich die Patienten vor allem durch Fatigue und Depression besonders eingeschränkt. Dieser Artikel zeigt mehrere Zugänge zu erfolgreichen Behandlungsstrategien, einschließlich eines homöopathischen Arzneimittels.

\section{Schlüsselwörter}

Long-COVID, Leberfeuer,Yin-Yang-Ausgleich, MittagMitternacht, Basisschwäche, Acidum picrinicum

\section{Summary}

The term Long-COVID includes persisting symptoms 4 weeks after infection. As affecting as they are, the patients have to endure cough and dyspnea, while above all fatigue and depression are the leading symptoms in Long-COVID suffering. Several approaches to a successful treatment including a homeopathic remedy are shown up in this article.

\section{Keywords}

Long-COVID, liver fire, Yin-Yang balancing, middaymidnight, basic weakness, acidum picrinicum
D er Begriff „Long-COVID“ ist kein medizinischer Fachbegriff im engeren Sinne. Er hat sich aber aufgrund der Praktikabilität in unserem Sprachgebrauch etabliert und fasst jene Symptome zusammen, die im Anschluss an eine COVID-Infektion bestehen bleiben oder neu auftreten und in Zusammenhang mit der vorangegangenen COVID-Infektion gebracht werden können.

Das National Institute for Health and Care Excellence (NICE) schlägt die in Tab. 1 aufgefuihrte Differenzierung derVerlaufsstadien vor.

Somit umfasst Long-COVID alle Symptome, die sich 4 Wochen und länger nach der Infektion zeigen.

Laut RKI sei bei milder COVID-19-Erkrankung rund jeder 10. länger als 4 Wochen betroffen und $40 \%$ der in der Klinik behandelten Patienten benötigen längerfristig Unterstützung [1].
Einer multizentrischen Studie aus London (Thorax 2021; 76: 396-398) zufolge, die 384 Patienten 54 Tage nach Krankenhausentlassung in einem Follow-up erfasst hat, berichteten $53 \%$ von persistierender Kurzatmigkeit und 34\% von Husten. Die Symptomatik von 69\% der

\begin{tabular}{|c|c|}
\hline $\begin{array}{l}\text { Zeitraum nach } \\
\text { Erkrankungsbeginn }\end{array}$ & Stadium \\
\hline 0 bis 4 Wochen & akutes COVID-19 \\
\hline 4 bis 12 Wochen & $\begin{array}{l}\text { anhaltendes symptomati- } \\
\text { sches COVID-19 }\end{array}$ \\
\hline ab 12 Wochen & Post-COVID-Syndrom \\
\hline
\end{tabular}


Patienten war einem Fatigue-Syndrom zuzuordnen und 14,6\% litten an einer Depression [2].

Um unseren Long-COVID-Patienten mit Akupunktur helfen zu können, müssen wir die Erkrankung zunächst aus dem traditionellen Blickwinkel betrachten. Auch wenn die Lunge oder das respiratorische System im Allgemeinen primär betroffen sind, ist aus traditioneller Sicht eigentlich ein anderes Organ für den Krankheitsverlauf zuständig: die Leber.

Es scheint, als würde COVID-19 eine besondere Affinität zum Yang der Leber besitzen und dieses noch weiter verstärken. Es handelt sich also um Leberfeuer, das, als mobilste Energieform überhaupt, grundsätzlich in jedes Organsystem hineinbrennen und dieses verletzen kann. Daher tritt diese Erkrankung auch auf so vielfältige Art und Weise in Erscheinung. Zusätzlich sucht sich das Leberfeuer immer den Weg des geringsten Widerstands, stört also in Organsysteme hinein, die in einer (konstitutionellen) Schwäche sind.

\section{Warum so häufig in die Lunge?}

Die Lunge, im Kontrollzyklus die Großmutter der Leber (Metall schneidet Holz), kann man, so sie nicht ohnehin das Organ der konstitutionellen Basisschwäche ist, in unserem Kulturkreis als durchaus geschwächt ansehen. Leben wir doch in einer Welt, in der alles ,,aus dem Takt" geraten $\mathrm{zu}$ sein scheint. Und darunter leidet natürlich am meisten unser Rhythmusgeber, die Lunge. Allzu oft wird die Nacht zum Tag gemacht. Oft wird bis spät in die Nacht gearbeitet und das Schlafdefizit kann tagsüber nicht ausgeglichen werden. Das führt auf Dauer zu einem Yin-Mangel. Denn nur der Nachtschlaf versorgt uns mit der nötigen Energie von Yin-Qualität. Ebendiese ist jedoch notwendig, um die Lunge vor Leberfeuer zu schützen. Hinzu kommen zahlreiche andere lungenschwächende Faktoren wie Umweltgifte, Rauchen oder Emotionen wie (anhaltende) Trauer, Traurigkeit und Melancholie. Es mag also wenig verwundern, dass die Lunge sich schwer bis kaum erholen kann und auch noch lange nach durchgemachter Infektion symptomatisch bleibt. Kurzatmigkeit und Husten zählen zu den häufigsten Symptomen bei Long-COVID und sind Ausdruck des hochdrängenden Leberfeuers, das die Lunge in ihrer absenkenden Funktion behindert.

Noch häufiger als Lungensymptome zeigt sich bei Long-COVID jedoch das Fatigue-Syndrom. Eine Erkrankung geprägt von bleierner Müdigkeit, begleitet von vielfältigsten Symptomen praktisch aus allen Funktionskreisen. Hier sei nochmals erwähnt, dass das Leberfeuer die mobilste Energieform ist und den Weg des geringsten Wider- stands sucht, der meist in die Lunge (Kurzatmigkeit, Husten), aber auch ins Herz (Palpitationen, Schlafstörungen) oder die Milz (Verdauungsstörungen, entzündliche Prozesse in Magen und Darm) führt.

Insgesamt bietet sich das Bild der sogenannten „Mangel-Hitze“. Denn Ursache für das pathognomonische Erschöpfungsgefuihl ist ein Yin-Mangel der Niere, der auch jeder weiteren Erscheinungsform von Long-COVID zugrunde liegt. Wie bereits erwähnt, führen die meisten Menschen in unserem Kulturkreis einen besonders Yinkonsumierenden Lebensstil, wodurch der kühlende, feuerlöschende Aspekt der Niere geschwächt wird. Das allein begünstigt schon das Auflodern des Leberfeuers und dessen Übergreifen auf andere Organsysteme. Wenn nun durch eine COVID-Erkrankung das Leberfeuer noch mehr angeheizt wird, entsteht ein massiver Yang-Überschuss, der enorm viel Yin verbraucht. Das kostet „Substanz" und die viel beschriebene bleierne Müdigkeit ist die Folge.

Abgeleitet von dieser traditionellen Betrachtungsweise ergeben sich nun auch die Behandlungsmöglichkeiten von Long-COVID mithilfe der Akupunktur, je nach Ausbildungsstufe.

Als Goldstandard gilt grundsätzlich der RAC zur Punktsuche.Alternativ kann aber natürlich das Punktsuchgerät verwendet werden oder es werden anhand der Druckschmerzhaftigkeit ,aktive“ Punkte identifiziert. Auch wenn das Punktekonzept immer individualisiert erstellt werden sollte, gilt es in jedem Fall, das Feuer zu löschen und den Yin-Aspekt des jeweilig geschwächten Organs zu stärken.

$\mathrm{Zu}$ prüfen sind daher besonders:

- die Sedierungspunkte - stärken besonders den YinAspekt des jeweiligen Organs,

- die Quellpunkte - leiten das Yin in das jeweilige Organsystem ein,

- die Alarmpunkte - bringen ebenso vermehrtYin in das Organsystem ein,

- die antiken Wasserpunkte - eignen sich besonders zum „Feuerlöschen“,

- KG 4 - als übergeordneter Yin-stärkender Punkt, der mit allen Sedierungspunkten in Verbindung steht.

Zur Behandlung kann Körper- oder Ohrakupunktur gewählt werden. In jedem Fall empfiehlt sich dieVerwendung von Dauernadeln, um eine Wirkungsverlängerung zu erreichen.

Eine besonders effiziente Behandlungsmöglichkeit in der Körperakupunktur bietet, unter Einbeziehung kyber- 

Herz: Amethyst
Lunge: Chrysopras
Milz: Beryll
Leber: Smaragd
Nieren-Yang: Chrysolith (Fr.: $16800 \mathrm{~Hz}$ )
Nieren-Yin: Diamant (Fr.: $8400 \mathrm{~Hz}$ )
Magen: Hyazinth
Galle: Karneol

Abb. 1: Den jeweiligen Organen zugeordnete Steinessenzen (Quelle: Manfred Reininger)

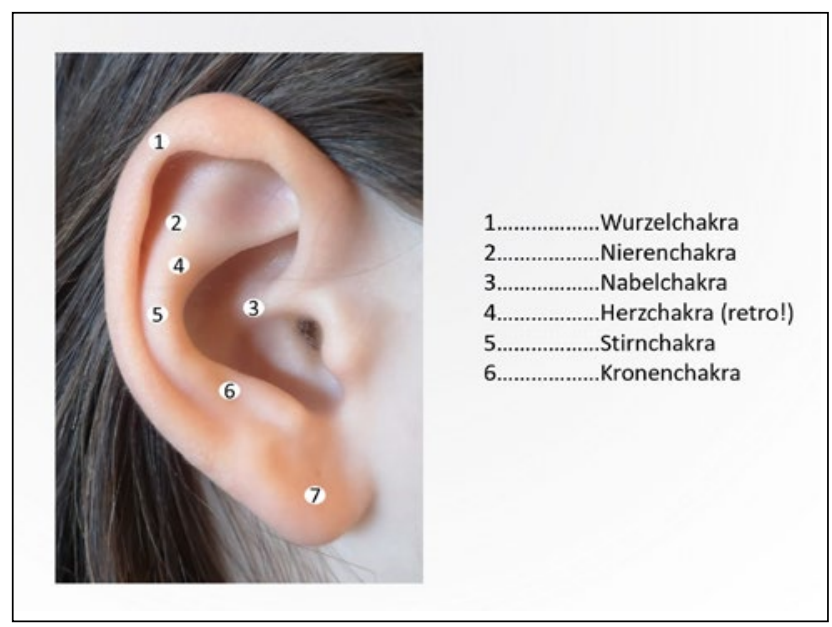

Abb. 2: Chakrenpunkte am Ohr nach Prof. Bahr (Quelle: Sandra Leeb)

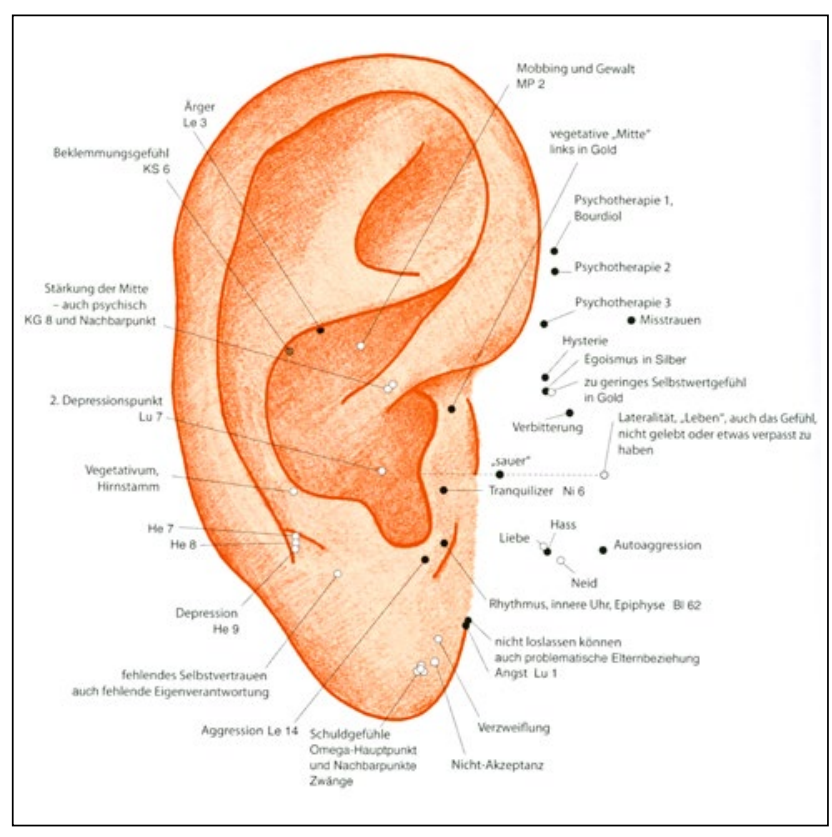

Abb. 3: Psychische Punkte nach Prof. Bahr

(Quelle: Ohrkarte der psychischen Punkte von Prof. Bahr) netischer Gesetzmäßigkeiten, die Regel Mittag-Mitternacht. Für diese gilt:

- Verbindung zweier Meridiane, deren chronobiologisches Maximum 12 Stunden auseinander liegt,

- Steuerung über die jeweiligen Luo-Punkte,

- jeweils ein Yin- und ein Yang-Meridian,

- jeweils ein Meridian oben, einer unten.

So wird ein intensiverYin-Yang-Ausgleich erzielt. Dieser läuft über die Mitte, die durch die große Energieverschiebung in Relation ,zu eng“ wird und daher unbedingt mitbehandelt werden muss (KG 12).

Das jeweilige Meridianpaar wird mit Lexotanil gefunden, das einerseits resonant ist auf MP 21, das sogenannte „Große Luo" und infolgedessen ebenso resonant ist auf die Luo-Punkte aller Meridiane. Daraus ergeben sich folgende Meridianbeziehungen:

\section{Dominante Seite - nicht dominante Seite:}

- $\mathrm{Lu} 7$ - Bl 58

- Di 6 -Ni 4

- Ma 40 - KS 6

- MP 4-3E 5

- He 5 - Gb 37

- Dü 7 - Le 5

Die Luo-Punkte auf der dominanten Seite werden tonisierend, auf der nicht dominanten Seite sedierend gestochen.

Ausnahme: MP 4 (tonisierend auf der nicht dominanten Seite) - 3E 5 (sedierend auf der dominanten Seite).

Und nicht vergessen: KG 12!

Hinzu kommt noch ein Blockadepunkt, der ebenfalls mit der Lexotanil-Ampulle im Bereich des Körperstamms gefunden wird.

Aus den obigen Überlegungen ergibt sich weiters, dass innerhalb der Long-COVID-Therapie der Basisschwäche (= Weg des geringsten Widerstands für das Leberfeuer) eine besondere Bedeutung zukommt. Diese sollte also aufgedeckt und behandelt werden.

Manfred Reininger hat hierzu bereits 2019 eine besonders effektive Behandlungsstrategie vorgestellt:

- Das betroffene Organ wird mit der Self-heal-Frequenz $4625 \mathrm{~Hz}$ (Bahr) im Diagnosemodus gesucht.

- Die Meridianfrequenz des gefundenen Organs wird peripher aufgelegt.

- Mit der entsprechenden Steinessenz (siehe unten) werden 5 Punkte gefunden:

1. der jeweilige Organpunkt (Abb. 1),

2. ein Chakrenpunkt (Abb. 2), 


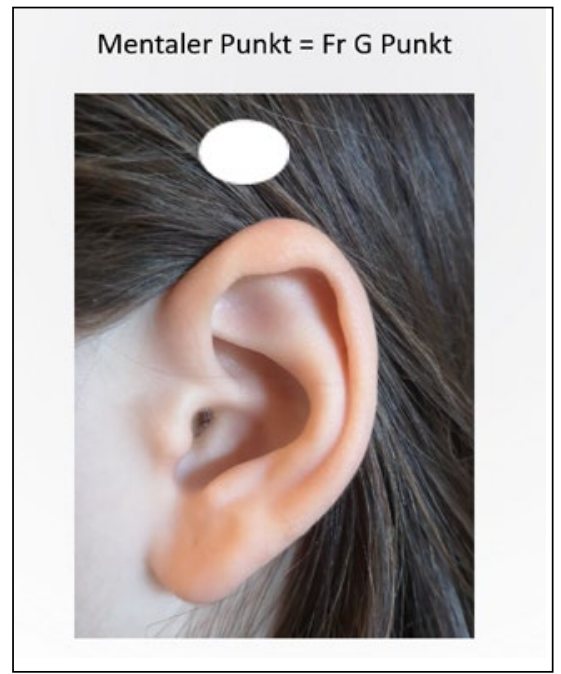

Abb. 4: Punkt der Mentalebene (Quelle: Sandra Leeb)

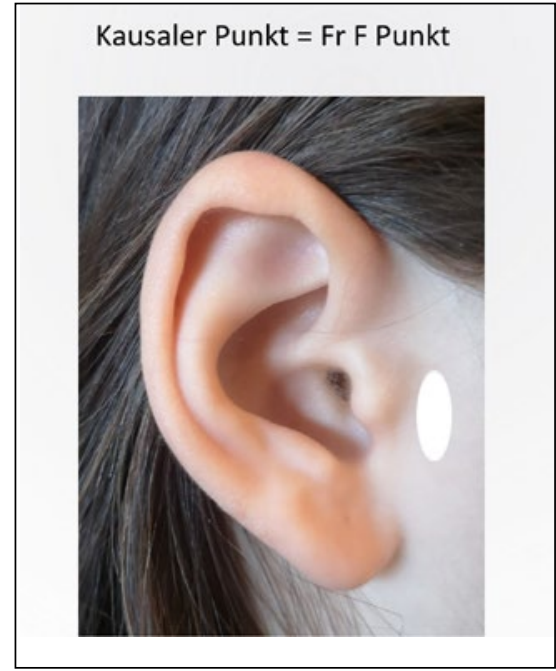

Abb. 5: Punkt der Kausalebene (Quelle: Sandra Leeb)

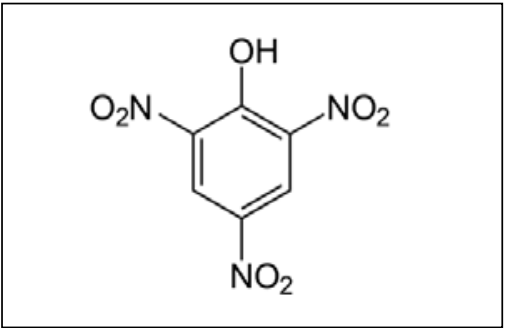

Abb.6: Strukturformel Pikrinsäure (Quelle: https://de.wikipedia.org/wiki/ Datei:Pikrins\%C3\%A4ure.svg)
3. ein psychischer Blockadepunkt (Abb. 3),

4. Punkt der Mentalebene (nicht dominant, liegt der Zone G gegenüber) (Abb. 4),

5. Punkt der Kausalebene (dominant, liegt der Zone F gegenüber (Abb. 5).

Ergänzend zu den Behandlungsmethoden mit der Akupunktur, hier noch ein kleiner Ausflug in die Homöopathie: Bisherigen Beobachtungen zufolge, hat sich Acidum picrinicum als sehr hilfreich in der Behandlung von COVID-Symptomen sowohl im akuten Stadium als auch in der Long-COVID-Behandlung erwiesen. In diesem Fall kann jedoch zusätzlich die Gabe der spezifischen oder einer miasmatischen Nosode erforderlich sein.

Hier einige Überlegungen der Homöopathin Ruth Svetitsch: Pikrinsäure ist eine explosive Substanz, die bereits an der Luft mit starker Rauchentwicklung verbrennt. Hier der Bezug zu den Atemwegen, da die Symptomatik eines schweren COVID-Krankheitsverlaufs der eines toxischen Lungenödems nach Inhalation einer konzentrieren Säure sehr ähnlich ist.

Die chemische Struktur des Pikrinsäuremoleküls (Abb. 6) enthält Elemente, die in den ersten beiden Reihen des Periodensystems liegen. Hier geht es ganz allgemein um das Thema Leben und Überleben, sowie um ganz basale Bedürfnisse, die allen Menschen gemeinsam sind.

- in der ersten Reihe Wasserstoff $\mathrm{H}$ - Thema Existenz,

- in der zweiten Reihe - Thema öffnen/schließen: Kohlenstoff C (hinein oder hinaus?), Stickstoff N (hinaus) und Sauerstoff (um jeden Preis, ohne Rücksicht auf andere).

Die Homöopathen Rajan Sankaran und Elizabeth Adalian weisen zusätzlich auf die Themen intellektuelle Überlastung, Überarbeitung und Burn-out sowie (alte) Traumatisierungen im Arzneimittelbild von Acidum picrinicum hin [3].

Die Wahl des Arzneimittels kann zunächst über dem „Gutpunkt“ KG 9 mittels RAC bestätigt werden, wobei die richtige Potenz über allen der vier Hauptenergiepunkten einen RAC ergeben muss: Yin-Tang, LG 20, KG 8, LG 4 (Optimum nach Prof. Bahr).

\section{Literatur}

[1] (Deutsches Ärzteblatt 49/2020)

[2] https://thorax.bmj.com/content/76/4/396

[3] New Homeopath, Summer 40:2

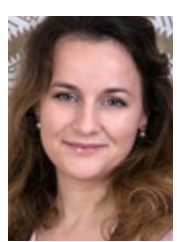

Dr. Sandra Leeb

Ärztin für Allgemeinmedizin, Notärztin

Ö̈̈K-Diplom Akupunktur, Ö̈̈K-Diplom Homöopathie

Praxis für Komplementärmedizin und Aurikulomedizin

1050Wien,dr-leeb@aurikuloaustria.at 